\title{
High-Resolution NMR Study on Hydrogen Bondings in Polyether Polyurethane Zwitterionomers
}

\author{
Guang Yang, Qun ChEN, Yuanshen Wang, and Xuewen Wu \\ Analytical Center, East China Normal University, Shanghai 200062, \\ People's Republic of China
}

(Received April 12, 1996)

\begin{abstract}
Hydrogen bondings formed in polyether-polyurethane zwitterionomers based on 4,4'-diphenylmethane diisocyanate (MDI), methyl diethanolamine (MDEA), and polytetramethylene oxide glycol (PTMO) were studied in concentrated solutions with high resolution ${ }^{1} \mathrm{H}$ NMR spectroscopy. By comparing with the results of a previous wide line NMR study, a direct relationship was found between the degree of phase separation and the amounts of various kinds of hydrogen bondings formed in the system. The formation of hard segment micelles in concentrated solution is proved and high resolution NMR is demonstrated to be a powerful tool to study the morphology of the multiphase polymers.

KEY WORDS Hydrogen Bonding / Polyether Polyurethane Zwitterionomer / ${ }^{1} \mathrm{H}$ Nuclear Magnetic Resonance /
\end{abstract}

Polyurethane elastomers are $(\mathrm{AB})_{n}$-type block copolymers. One block, the "hard segment," is normally composed of aromatic diisocyanate chains which is often extended with low molecular weight diols. The other block is usually polyether, polyester diol and is termed "soft segment" since the $T_{\mathrm{g}}$ of this block is normally below service temperature. Certain degree of phase separation between these two blocks is essential to the mechanical properties of the material. Through changing the components, molecular weight of either the hard segment or the soft segment, we can influence the phase separation of the material, which in turn changes the properties of the material. For the importance of the material, many studies have been focused on this field. ${ }^{1-4}$

One way to influence the properties of the polyurethane elastomers is ionization, ${ }^{4}$ that is, the incorporation of ionic groups into the hard segments. It has been found that ionization changes both the polarity and regularity of the hard segments and therefore influences the phase separation of polyether polyurethanes greatly.

The formation of hydrogen bonds also bears great influence on the phase separation of the polyurethane elastomers. ${ }^{5-8}$ Polyurethane elastomers whose soft segments have a higher hydrogen bond affinity tend to form hard segment-soft segment hydrogen bond and therefore is less phase separated. ${ }^{5.6}$ Due to the importance of the hydrogen bonding in the system, it has been carefully studied by various methods including FTIR, NMR etc. ${ }^{9-11}$

Previous NMR studies have proved there exists a similarity between the morphology of the polymer in concentrated solution and that of the polymer in bulk. ${ }^{12}$ So high resolution NMR have been employed to study the high order structure of the polymer, including phase separation, interactions between different segments etc. ${ }^{9-11}$ When observing the high resolution ${ }^{1} \mathrm{H}$ NMR spectrum of polyurethane zwitterionomers, the number of $\mathrm{NH}$ peaks observed is often larger than that expected from the chemical structure of the polymer. Some authors assigned these extra peaks to the secondary structures of the urethane ${ }^{13}$ while others assigned them to the formation of different kinds of hydrogen bondings..$^{9-11}$ Therefore there arises a need of careful assignment of these NH peaks before using these peaks to study the hydrogen bondings in the system. In this work, $\mathrm{NH}$ peaks in high resolution ${ }^{1} \mathrm{H}$ NMR spectra are carefully assigned and used to investigate the hydrogen bondings in the polyether polyurethane zwitterionomers and their variance with the degree of ionization. The influence of the hydrogen bondings to the phase separation is discussed and the results are compared with those obtained in solid NMR study.

\section{EXPERIMENTAL}

\section{Samples}

Polyether polyurethanes are based on polytetramethylene oxide glycol (PTMO) with a molecular weight of 1000 and 4,4'-diphenylmethane diisocyanate (MDI) extended with methyl diethanolamine (MDEA). Ratio of contents of three components is MDI : MDEA : PTMO = $3: 2: 1$. Ionization are incorporated into MDEA with addition of controlled amount of $\gamma$-propane sultone. ${ }^{14}$ Samples with different degree of ionization are polymerized in a batch and then ionized with different amount of $\gamma$-propane sultone. Four samples named PUA, PUB, PUC, and PUD, respectively, are used and their chemical structures are shown in Scheme 1.

\section{NMR Experiments}

High resolution NMR are performed on a Bruker MSL-300 spectrometer with ${ }^{1} \mathrm{H}$ resonance frequency at

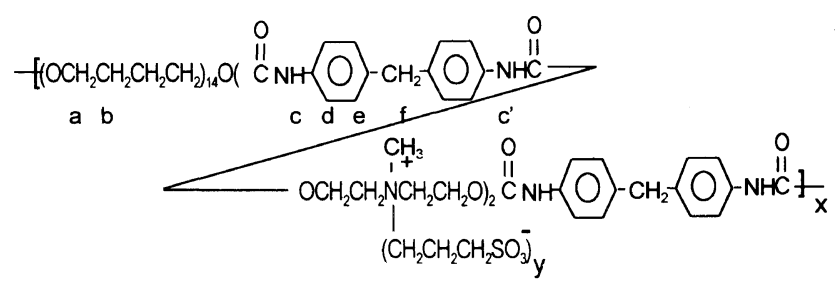

Scheme 1. Chemical structure of polyetherpolyurethane zwitterionomers. Where $y$ represents degree of ionization, which are $0,0.35$, 0.65 , and 0.95 for PUA, PUB, PUC, and PUD, respectively. 
300.13 MHz. Samples for NMR experiments are dissolved in deuterated $N, N^{\prime}$-dimethylformamide (DMF$d_{7}$ ). The concentration used is over $20 \% \mathrm{w} / \mathrm{v}$. ${ }^{1} \mathrm{H}$ Spectra are acquired with standard one pulse methods. Flip angle is $30^{\circ}$ while delay time is $2 \mathrm{~s}$. Typically 256 scans are accumulated in variable temperature experiments and 1200 scans are accumulated to get the standard ${ }^{1} \mathrm{H}$ spectra at room temperature. Chemical shifts are determined according to the low field peak of DMF $(8.01 \mathrm{ppm})$. Temperature is controlled with a Bruker VT-1000 temperature control unit. Deviation of temperature is $\pm 1 \mathrm{~K}$.

\section{RESULTS AND DISCUSSION}

\section{Assignment of High-Resolution ${ }^{1} H$ NMR Spectra}

High resolution ${ }^{1} \mathrm{H}$ NMR spectra of PUB and the assignment of main peaks are shown in Figure 1. Peaks are assigned referencing to the works of Wang et al. ${ }^{15}$ and Natansohn et al. ${ }^{13}$ It is noticeable that six NH peaks are observed in the range of $8.0-10.5 \mathrm{ppm}$ and those NH peaks vary dramatically with the degree of ionization (Figure 2). For convenience of discussion, from low field to high field, these peaks are labeled A, B, C, D, E, and $F$, respectively.

Though there exist two kinds of $\mathrm{NH}$ groups (labeled as $c$ and $c^{\prime}$ in Scheme 1) in the polymer, the difference in chemical structure is five chemical bonds away from the NH group, which is normally too far to have significant influence on the chemical shifts of $\mathrm{NH}$. Therefore all six NH peaks must be assigned to secondary structures (Scheme 2) suggested by Natansohn ${ }^{13)}$ or formation of different kind of hydrogen bondings.

Secondary structures occur in the process of polymerization of the polyether polyurethane. Since all samples studied are polymerized in a batch before ionized separately, the integral of the NH peaks assigned to secondary structures must keep constant in all samples. It can be seen in Scheme 2 that secondary reactions bring about branching and crosslinking which will make the sample insoluble when the content of secondary struc-

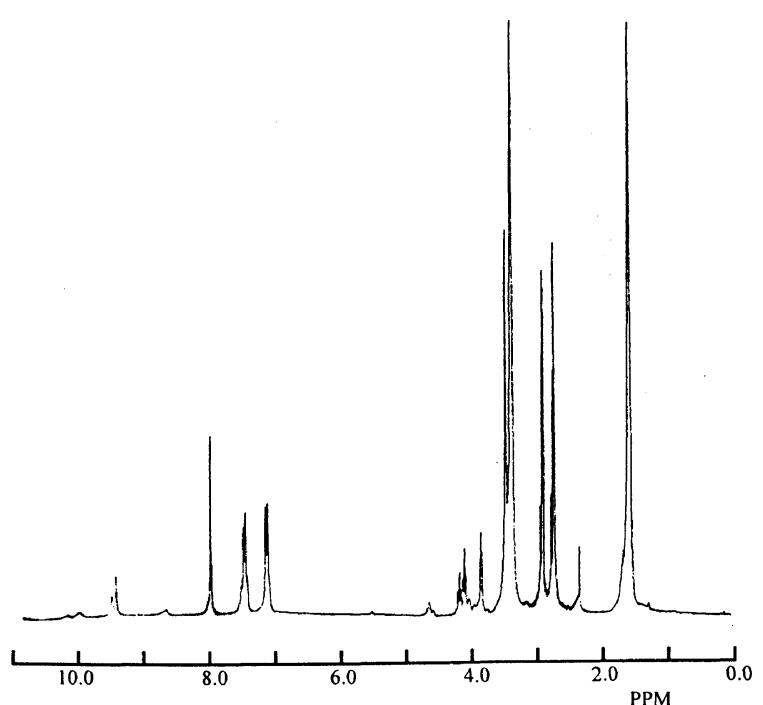

Figure 1. ${ }^{1} \mathrm{H}$ NMR spectrum of PUB. Assignment of main peaks is as follows (see Scheme 1 for chemical structure): a, $3.38 \mathrm{ppm}$; b, $1.66 \mathrm{ppm}$; c, $8.5-10.2 \mathrm{ppm}$; d, $7.50 \mathrm{ppm}$; e, $7.14 \mathrm{ppm}$; f, $3.85 \mathrm{ppm}$; water, $3.50 \mathrm{ppm}$. ture is high enough.

Thus, a peak of secondary structure must satisfy following two criteria: 1) the relative integral of the peak does not change with the samples; 2) the integral of the peak must be relatively small so as not to produce crosslinking in the samples. From relative integral of all

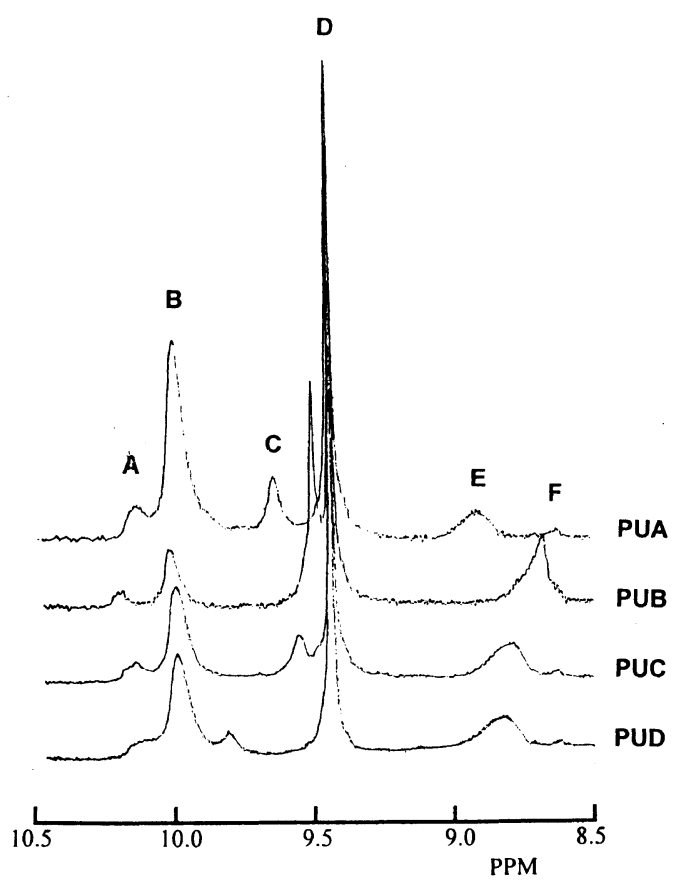

Figure 2. $\mathrm{NH}$ peaks of polyether polyurethane zwitterionomers. From upper to lower are spectra of PUA, PUB, PUC, and PUD, respectively.
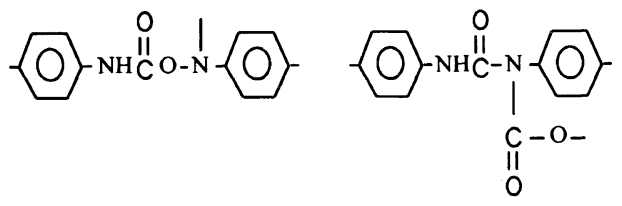

Structure I

Structure II

Scheme 2. Secondary structures that may be formed in the process of polymerization.

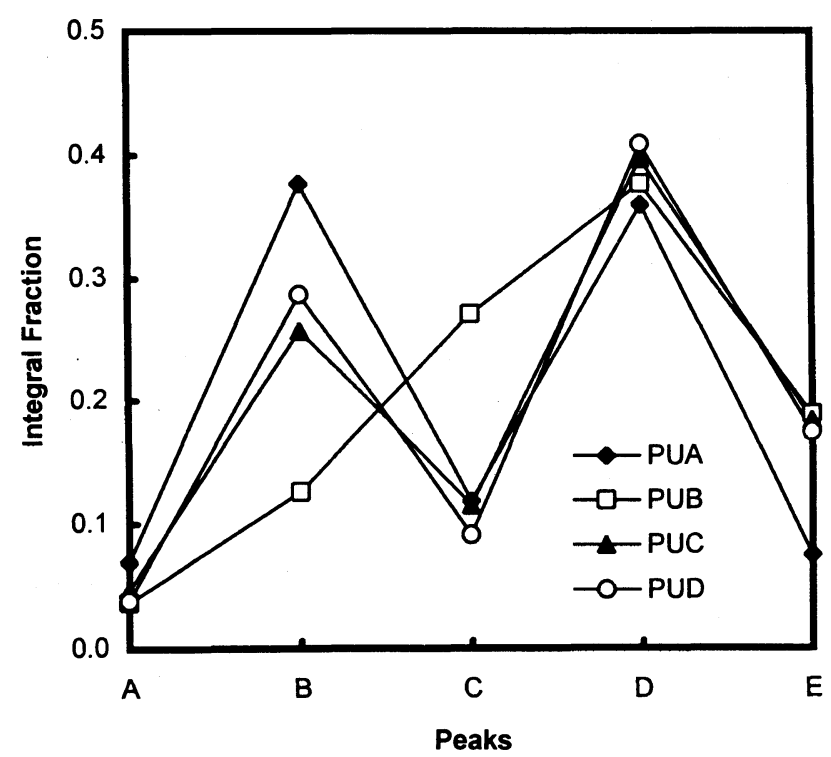

Figure 3. Relative integral of $\mathrm{NH}$ peaks of polyurethane zwitterionomers. Peak $\mathrm{F}$ is too small to be integrated precisely, so it is not included here. 
NH peaks of four samples (Figure 3), it can be found only peak $A$ and peak $F$ satisfy the above condition, so they may be assigned to NH group in secondary structure. According to their chemical shifts, peak $\mathrm{A}$ and $\mathrm{F}$ can be assigned to structure I and II in Scheme 2, respectively.

The remaining NH peaks can only be explained by the formation of different kinds of hydrogen bonds. The following groups may form hydrogen bonds with the $\mathrm{NH}$ : oxygen in PTMO, carbonyl group in hard segment, nitrogen in MDEA, carbonyl group in solvent, and oxygen in ionization group. The carbonyl group in hard segment may also form intramolecular hydrogen bond with the adjacent $\mathrm{NH}$ group. For the requirement of bond direction, this intramolecular hydrogen bond is not as strong as other kinds of hydrogen bonds formed in the system.

It can also be found from Figure 3 that:

1) $\mathrm{NH}$ groups form no hydrogen bonds with the nitrogen atoms in MDEA. If a peak is representing $\mathrm{NH}$ hydrogen-bonded to the nitrogen atoms in MDEA, its relative intensity in PUD should be less than $2 \%$, which is calculated assuming that all non-ionized nitrogen atoms in MDEA are hydrogen-bonded to NH groups;

2) The fact that all the NH peaks appear in the spectra of the non-ionized sample PUA excludes the possibility of NH forming hydrogen bondings with the ionization groups.

Taking the above discussions and the strength of the hydrogen bondings in mind, peak $\mathrm{B}, \mathrm{C}, \mathrm{D}$, and $\mathrm{E}$ are assigned to the $\mathrm{NH}$ hydrogen-bonded to carbonyl group in hard segment (intermolecular), oxygen in PTMO, solvent, and adjacent carbonyl group, respectively.

On the basis of the above assignment, it can be observed from Figure 3 that the amount of intersegmental hydrogen bonds formed between $\mathrm{NH}$ and carbonyl groups in hard segments (peak B) changes dramatically with the degree of ionization. The amount of these inter-hard-segmental hydrogen bonds is the largest in the non-ionized PUA, and the lowest in PUB, whose degree of ionization is $35 \%$. From a previous variable temperature solid NMR study on this series of samples, ${ }^{16,17}$ it is known that the hard segments in PUA show certain degree of regularity or crystallinity that gives PUA the largest degree of phase separation in four samples. Obviously, this regularity of the hard segments in PUA is retained in the concentrated solutions, because the amount of the inter-hard-segmental hydrogen bonds is the highest in four samples.

In PUB, the integral of peak B decreases while the integral of peak $\mathrm{C}$, which representing the hydrogen bonding interaction between hard and soft segments, increases. This observation is also in good agreement with that observered in solid NMR: Initial incorporation of ionization groups destroys the regularities of the hard segment and increases the compatibility of hard and soft segments.

Further ionization increases the hard segment cohesion and difference of the polarity between hard and soft segments, therefore helps to regain certain degree of phase separation in PUC. So in PUC, the intensity of peak $B$ increases while the intensity of peak $C$ decreases. It can also be observed from Figure 3 that the ratios of different hydrogen bondings in PUC and PUD are almost the same. This result is also consistent with that obtained from solid NMR study: when the degree of ionization exceeds $65 \%$, further incorporation of ionization groups into hard segments has little influence on the phase separation of the samples.

From about discussion, it can be known that the change of amount of various kinds of hydrogen bonds formed in the system reflected exactly the comatibility of the polymer in bulk. The inter-segmental interactions in concentrated solution resemble that in bulk. It can also be seen from Figure 3 that the relative amount of hydrogen bonds formed with solvent is less than $40 \%$ in all samples, which suggests that certain part of hard segment have little contacts with the solvent. The above observations can only be explained by the existence of the hard segment micelles in the concentrated solutions. In these micelles, solvent has little influence on the chain packing and inter-segmental interactions which resemble those of polymers in bulk. It is also known that certain parts of the hard segments are well dissolved in the solvent, which is somewhat different from the results of Lu et al. ${ }^{11}$

\section{Results of Variable Temperature ${ }^{1} H$ NMR}

To further verify above results, variable temperature ${ }^{1} \mathrm{H}$ NMR spectra of four samples are measured over a temperature range of $290-380 \mathrm{~K}$, at a $10 \mathrm{~K}$ interval. Since chemical shifts of peaks other than $\mathrm{NH}$ and $\mathrm{H}_{2} \mathrm{O}$ peaks do not change with the temperature, only chemical shifts of NH groups are plotted against temperature in Figure 4 , taking PUA as an example. The changes of chemical shifts of NH peaks of all samples are listed Table I.

As the temperature increases, all $\mathrm{NH}$ peaks and $\mathrm{H}_{2} \mathrm{O}$ peak shift to high field while their relative intensities keep constant. All NH groups are in an exchange process between hydrogen-bonded and non-hydrogen-bonded states. If this exchange process is not quick enough, two separate peaks will be observed: one for hydrogenbonded NH and one for non-hydrogen-bonded NH.

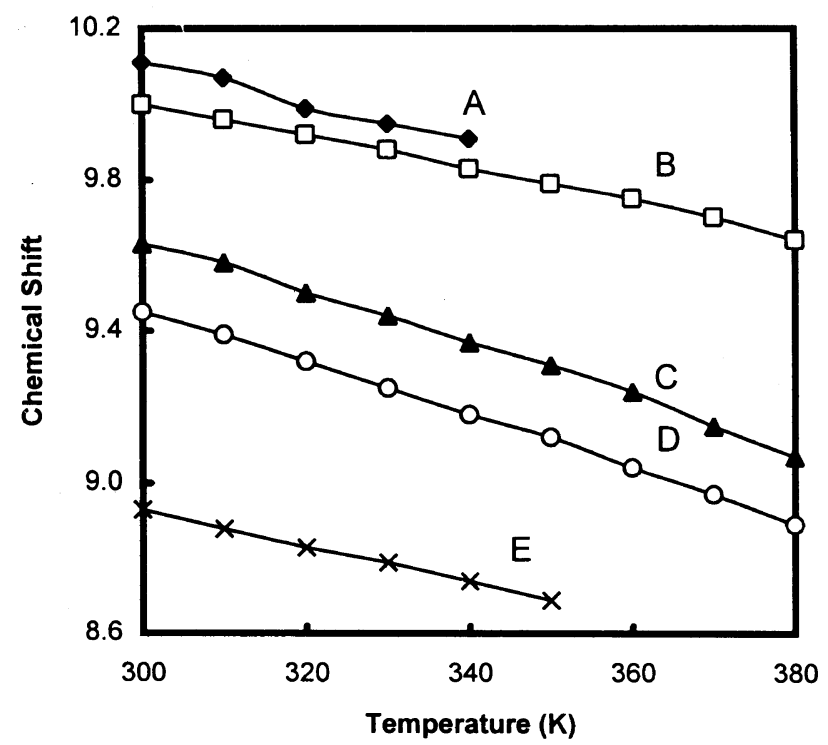

Figure 4. Change of chemical shift of NH peaks of PUA with temperature. Peak F is hardly observable in the variable temperature experiments. 
Table I. Variation of chemical shifts of NH peaks with the temperature ${ }^{\mathrm{a}}$

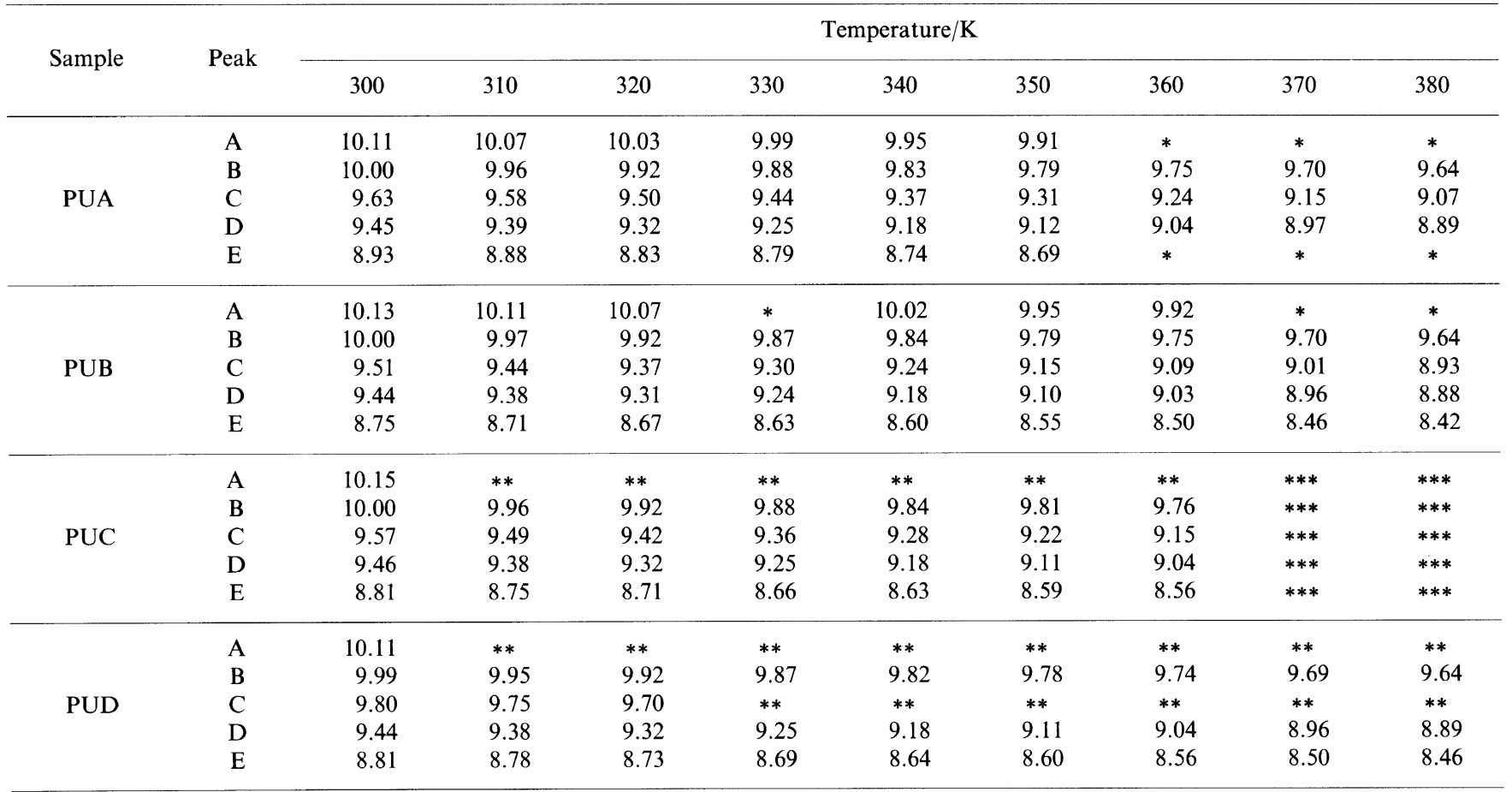

${ }^{a}$, not accessible for its poor $\mathrm{S} / \mathrm{N}$. $* *$, not accessible for overlapping with adjacent peak. ***, not measured for poor magnetic field homogeneity at higher temperature.

Note: Peak F is so small that it is difficult to observe in most variable temperature experiment, so its chemical shifts is not included in this table.

With the increase of the temperature, the exchange process becomes faster, which in turn makes two peaks come closer. The peak representing non-hydrogenbonded NH should move to low field under such circumstances. Since no NH peaks are found to move to low field with the increase of tempreature, it is clear that the exchange process is fast enough to give only one peak for one kind of hydrogen bonds.

NH peaks can be divided into two groups by the change rate of the chemical shift with temperature. The first group includes peak $\mathrm{A}, \mathrm{B}$, and $\mathrm{E}$, the change rate of which is about $0.04-0.05 \mathrm{ppm} / 10 \mathrm{~K}$. The second group includes peak $\mathrm{C}$ and $\mathrm{D}$, whose change rates are approximately $0.08 \mathrm{ppm} / 10 \mathrm{~K}$. As a result of this difference of chemical shift shifting rate, peak $\mathrm{C}, \mathrm{D}$, and $\mathrm{E}$ come closer with the increase of temperature, suggesting that there exists chemical exchange among these peaks. Peak $\mathrm{C}$ and D represent $\mathrm{NH}$ groups forming hydrogen bonds with soft segments and solvent respectively. For the mobility of soft segments and solvent, these two kinds of hydrogen bonds are easy to decompose and then form intramolecular hydrogen bonds (Peak E), so it is understandable that there exists an exchange among $\mathrm{C}$, $\mathrm{D}$, and $\mathrm{E}$. On the other hand, there is no exchange between peak B and other peaks, which indicates NH groups forming the inter-hard-segment hydrogen bonds are difficult to decompose. This result strongly supports our previous conclusion that there exist hard segment micelles in which the hard segments have limited contact with the solvent and therefore are relatively rigid.

\section{Results of Variable Concentration Experiments}

${ }^{1} \mathrm{H}$ NMR measurements are also carried out at different sample concentrations varying from $10-30 \%$ $\mathrm{w} / \mathrm{v}$. The variation of the concentration has no influence on either chemical shifts or the integrals of the $\mathrm{NH}$ peaks. It is understandable since the conformation of the polymer in the concentrated solution does not change with the concentration of the solution. This result also supports the existence of hard segment micelles in which the hard segment conformation is relatively stable.

\section{CONCLUSIONS}

Following conclusions can be drawn from above discussions:

1. The structure model for polyether polyurethane zwitterionomers can be visualized as follows: a portion of hard segments form micelles in which polymer chains have little contact with the solvent and retain a relative rigid conformation. Soft segments and the remaining of hard segments are extended or coiled in the solvent, linking the micelles together. Such a kind of structure can easily lead to the formation of a morphology described by Bead-Spring model ${ }^{18}$ when the samples are cast from concentrated solutions.

2. The results of high resolution NMR study about the hydrogen bonding interactions are consistent with the results of wide line ${ }^{1} \mathrm{H}$ NMR study on the morphology of the bulk polymers, so forming of hydrogen bondings may have great influence on the morphology of polyurethane zwitterionomers.

3. Solution NMR can be used to study factors influencing the morphology of the multiphase polymers due to its high resolution characteristics. Combining with the solid state NMR, it can be a powerful tool to probe the morphology of the multiphase polymers. 
Acknowledgments. This work is supported by Foundation of the Nation's Education Committee of China. The authors thank Prof. C. Z. Yang of Nanjing University for kindly providing the samples used in this study.

\section{REFERENCES}

1. S. L. Cooper and G. M. Estes, Ed., "Multiphase Polymers," Advanced Chemistry Series, 176, The American Chemical Society, Washington, D. C., 1979.

2. J. A. Miller, K. K. S. Hwang, and S. L. Cooper, J. Macromol. Sci., Phys., 22, 153 (1984)

3. J. W. C. Van Bogart, P. E. Gibson, and S. L. Cooper, J. Polym. Sci., Phys., 21, 65 (1983)

4. C. Z. Yang, T. G. Grasel, R. A. Register, and S. L. Cooper, J. Polym. Sci., Phys. Ed., 29, 581 (1991).

5. K. K. S. Hwang, S. B. Lin, S. Tsay, and S. L. Cooper, Polymer, 26, 947 (1984)
6. K. K. S. D. J. Hemker and S. L. Cooper, Macromolecules, 17, 307 (1984).

7. C. M. Brunette, S. L. Hsu, M. Rossman, W. J. MacKnight, and N. S. Schneider, Polym. Eng. Sci., 21, 668 (1981).

8. T. A. Speckhard, G. Ver Strate, P. E. Gibson, and S. L. Cooper, Polym. Eng. Sci., 23, 337 (1983).

9. X. Lu, Y. Wang, and X. Wu, Polymer, 33, 958 (1992).

10. X. Lu, Y. Wang, and X. Wu, Polymer, 35, 2315 (1994).

11. X. Lu, M. Hou, X. Gao, and S. Chen, Polymer, 35, 2510 (1994).

12. P. A. Mirau and F. A. Bovey, Macromolecules, 23, 4548 (1990).

13. A. Natansohn, M. Rutkowska, and A. Eisenberg, Polymer, 28, 885 (1985).

14. C. Z. Yang, K. K. S. Hwang, T. A. Speckhard, and S. L. Cooper, Macromol, Chem., 183, 651 (1983).

15. Y. Wang and M. Guo, Chinese. J. Polym. Sci., 1, 72 (1986).

16. G. Yang, Q. Chen, Y. Wang, X. Wu, and C. Yang, Chinese J. Polym. Sci., 12, 302 (1994).

17. G. Yang, Q. Chen, Y. Wang, C. Yang, and X. Wu, J. Mol. Struct., 323, 209 (1994)

18. D. C. Lee, R. A. Register, C. Z. Yang, and S. L. Cooper, Macromolecules, 21, 1005 (1988). 